\title{
Оценка характеристик
}

\section{процесса распространения социальных инноваций в Российской Федерации}

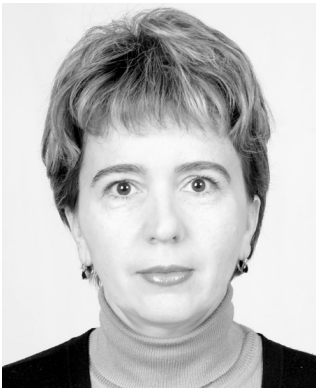

О. Г. Кантор,

к. Ф.-м. н., доцент, старший научный сотрудник o_kantor@mail.ru

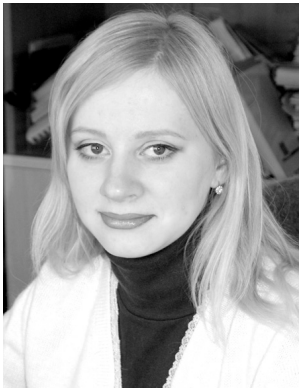

Ю. А. Кузнецова, к. э. н., старший научный сотрудник acanaria2005@yandex.ru

\section{Институт социально-экономических исследований Уфимского научного центра Российской академии наук}

Для России понятие «сочиальные инновации» является относительно новым с точки зрения как теоретикометодологического оформления, так и практического использования. В работе представлень результаты исследований характеристик процесса распространения социальных инноваций, проведенных с позиций традиционных и специфических подходов. Показано, что в Российской Федерации современная среда распространения сочиальных инноваций имеет «вязкий» характер, проявляющийся, в частности, в ограниченных возможностях распространения новшеств и проблемах их масштабирования. Практические шаги по регулированию процесса распространения социальных инноваций привели к созданию Агентства социальных инициатив - структуры, аккумулирующей сощиальные инициативы, определяющей направления и приоритеты развития и распространения сочиальных инноваций. Однако, учитывая новизну данного направления деятельности для нашего государства, особую значимость приобретает разработка специфических методов исследования инновационных процессов в сочиальной сфере, применение которых будет способствовать всестороннему анализу и повышению обоснованности прогнозных оценок. Одним из подходов к реализации сказанного является использование концепиии диффузии инноващий, в рамках которой авторами был предложен метод определения параметров модели Басса, осуществлены соответствующие расчеты и дана трактовка полученных результатов.

Ключевые слова: социальные инновации, инновационный проект, распространение, оценка, диффузия, инноватор, имитатор.

\section{Введение}

Социальные инновации представляют собой идеи, проекты, организации, направленные на удовлетворение потребностей общества и повышение качества жизни населения. Несмотря на тот факт, что инновационная проблематика исследований широко распространена среди российских ученых и специалистов, до сих пор не существует единого подхода к пониманию принципов и универсальных методов управления социальными инновациями. При этом следует признать, что сложившаяся социально-экономическая ситуация в Российской Федерации не позволяет не только внедрять и распространять, но и всесторонне развивать эффективные социальные технологии.

В настоящее время в России отсутствует целостная система управления инновациями в социальной сфере: функции управления организационно не структуриро- ваны, существующая институциональная структура не адаптирована под реализацию задач эффективного и результативного распространения социальных инноваций, отсутствует практика централизованного сбора и обработки информации о деятельности соответствующих организаций. К числу наиболее значимых показателей, используемых за рубежом для оценки социальных инноваций и не представленных в российской практике, относятся следующие:

- общее количество реализуемых и потенциальных инновационных проектов в социальной сфере;

- $\quad$ количество инновационных проектов по отдельным отраслям социальной сферы;

- $\quad$ количество инновационных проектов, перенятых для реализации из опыта лучших практик иных организаций;

- количество инициаторов инновационных проектов; 


\section{ИННОВАЦИОННАЯ ЭКОНОМИКА}

• количество благополучателей социальных инновационных проектов ${ }^{1}$ и др.

Само по себе исследование процессов распространения социальных инноваций призвано способствовать выявлению наиболее значимых и приоритетных направлений роста уровня их доступности, что, очевидно, влияет на качество жизни населения. В этой связи актуальным является задействование как традиционных, так и специфических методов изучения таких процессов.

\section{Состояние сферы социальных инноваций}

В настоящее время, единственным органом, действующим в целях оказания поддержки и содействия в решении многообразных системных проблем, в том числе, распространения социально значимых инициатив, является Агентство стратегических инициатив (АСИ). АСИ - автономная некоммерческая организация, образованная в 2011 г. Правительством Российской Федерации [4]. Под эгидой Агентства динамично расширяется региональная сеть Центров инноваций социальной сферы, целью деятельности которых является сопровождение социальных проектов малого и среднего бизнеса, а также проектов, разрабатываемых социально-ориентированными НКО. Организация центров инноваций социальной сферы (ЦИСС) проводится в соответствии с рекомендованной для их функционирования структурой (рис. 1), однако на сегодняшний день далеко не во всех регионах такие структуры реализованы в полной мере.

На сегодняшний день крупнейшим в России является Омский центр инноваций в социальной сфере. В начале своей деятельности под эгидой АСИ в 2012 г. Центр работал с шестью регионами России, но уже в 2014 г. - с 19-ю субъектами. В 2012 г. индивидуальные консультации получили 154 предпринимателя, в 2014 г. - 586. Примечательно, что благополучателями только 52 реализованных социальных проектов стали 16 тыс. человек. Отметим, однако, что реализация данных проектов носит узко адресный характер и не предполагает широкомасштабного распространения инновационных проектов.

Анализ процесса распространения социальных инноваций позволил в качестве основных идентифицировать следующие его негативные характеристики:

- отсутствие практики сбора какой бы то ни было статистической информации не только о результатах внедрения социальных проектов, но и ресурсов, задействованных на их выполнение, затрудняет оценку эффекта их реализации, масштабов распространения;

- неактивная политика тиражирования значимых социальных проектов, что сужает круг потенциальных благополучателей;

1 Центры социальных инноваций: CЕI (Вашингтон, США), Skoll Centre for Social Entrepreneurship (University of Oxford, Великобритания), Connecticut Center for Social Innovation (Коннектикут), Harvard Kennedy School of Government Social Impact Bond Technical Assistance Lab (SIB Lab, CШA), Centre for Social Innovation (Торонто), Lien Centre for Social Innovation (Сингапур) и др.

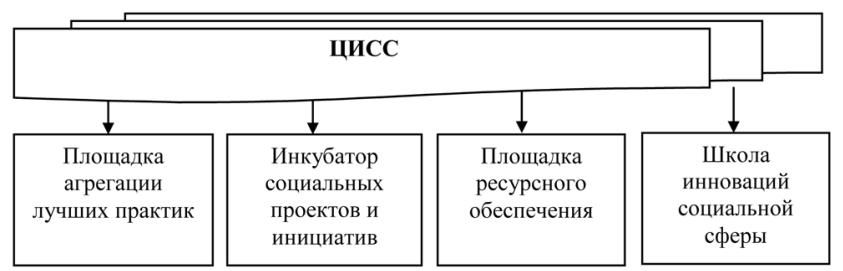

Рис. 1. Структура Центров инноваций социальной сферы Источник: составлено с использованием - «Сеть Центров инноваций социальной сферы. Проект АСИ по развитию социального предпринимательства в регионах» [4]

- $\quad$ недостаточная активность субъектов инновационного процесса: подавляющее количество инновационных проектов в социальной сфере реализуется инноваторами - участниками рынка, способными идти на риск при выборе новшеств, и лишь малая часть - имитаторами - теми, кто перенимает новшества по мере их проникновения на рынок.

В целом, среда распространения социальных инноваций в России имеет «вязкий» характер, проявляющийся в ограниченных возможностях их распространения, трудном масштабировании, сложности передачи схожим целевым группам, расположенным на иных территориях, что усугубляется скудным количеством статистических данных, не позволяющим осуществлять всесторонний анализ и оценку инновационных процессов. Так, АСИ раскрывает только 4 показателя, обеспечивающих возможность оценки деятельности организации в части развития социальных инновационных проектов, три из которых представлены в табл. 1.

Четвертый показатель - «Открыто центров социальных инноваций, кол-во регионов» - не следует рассматривать в качестве информативного в силу того, что уже к 2014 г. он достиг своего максимума: в 2012 г. количество регионов, участвующих в проекте по созданию центров социальных инноваций, составляло 7 ед., в 2013 г. их стало 43, в 2014 г. -83.

На сегодняшний день АСИ по сути является основной структурой, осуществляющей прогноз показателей в сфере социальных инноваций у нас в стране. Согласно его прогнозам за период 20122016 гг. количество социальных предпринимателей должно возрасти в 31 раз, а количество запущенных социально-предпринимательских проектов - в 87,5 раза. Однако, учитывая новизну данного направления деятельности для нашего государства, особую значи-

Таблица 1

Показатели для оценки деятельности АСИ в части развития социальных инновационных проектов

\begin{tabular}{|l|c|c|c|c|c|}
\hline Показатель & 2012 г. & 2013 г. & 2014 г. & 2015 г. & 2016 г. \\
\hline $\begin{array}{l}\text { Количество со- } \\
\text { циальных предпри- } \\
\text { нимателей, чел. }\end{array}$ & 500 & 5000 & 10000 & 12000 & 15500 \\
\hline $\begin{array}{l}\text { Количество запу- } \\
\text { щенных социально- } \\
\text { предприниматель- } \\
\text { ских проектов, ед. }\end{array}$ & 80 & 1000 & 5000 & 6800 & 7000 \\
\hline $\begin{array}{l}\text { Количество центров } \\
\text { инноваций в соци- } \\
\text { альной сфере, ед. }\end{array}$ & 4 & 8 & 15 & 15 & 17 \\
\hline
\end{tabular}

Источник: данные АСИ [4] 


\section{ИННОВАЦИОННАЯ ЭКОНОМИКА}

мость приобретает разработка специфических методов исследования инновационных процессов в социальной сфере, применение которых будет способствовать всестороннему анализу и повышению обоснованности прогнозных оценок.

\section{Метод исследования}

В рамках существующих подходов к исследованию инноваций одно из ключевых мест отводится концепции диффузии инноваций. Впервые диффузия инноваций была изучена французским социологом Жан-Габриэль де Тард, который в 1890 г. опубликовал работу с результатами исследования процессов перенимания и заимствования инноваций. Первая теоретическая модель диффузии инноваций, полученная с использованием имитационного подхода, была построена T. Хагерстрандом (1953 г.). Основные теоретические положения диффузии инноваций были сформулированы Э. Роджерсом на основе обобщения более 500 исследований в различных социально-экономических сферах (1962 г.) и на сегодняшний день составляют основу теории инноваций.

Диффузия инноваций заключается в распространении новшеств, в качестве которых могут выступать идеи, технологии, продукция и пр., являющиеся новыми для их потребителя в различных сферах деятельности государства: научной, технической, производственной, организационной. В ходе диффузионного этапа постепенно увеличивается количество потребителей новшества. Это может стать предпосылкой к формированию нового технологического уклада в обществе, а в случаях, когда обновлению подвергается значимая часть экономики государства, - и ее структурной перестройки.

Целесообразность исследования диффузии инноваций обусловлена эффективностью диффузионного процесса с позиций обеспечения становления государства как индустриально развитого и интегрированного в мировое экономическое пространство. Такие исследования актуальны как при изучении отдельных аспектов, например, диффузии технологических инноваций [3], способных обеспечить получение обоснованных выводов относительно системы трансферта технологий внутри страны, так и при изучении сферы инноваций в целом.

Изучение диффузионных процессов в инновационной сфере осуществляется при помощи специфических моделей, основное назначение которых состоит в изучении распространения новшества в рамках социальной системы с течением времени. Базовые модели для исследования диффузии инновационных процессов подразделяются:

1) на модели с учетом инновационного покупательского поведения;

2) модели с учетом имитационного покупательского поведения;

3) модели с учетом как инновационного, так и имитационного покупательского поведения.

Примером модели первой группы является модель Форта и Вудлока [6], которая была разработана для исследования эффектов от внедрения новых гастрономи- ческих товаров (1), моделей второй группы - модель Мэнсфилда (2):

$$
\begin{aligned}
& \frac{d F}{d t}=p(1-F), \\
& \frac{d F}{d t}=q\left(F-F^{2}\right),
\end{aligned}
$$

где $F$ - количество пользователей новшеством, выраженное в долях единиц; $p$ и $q$ - параметры, характеризующие, соответственно, степень проникновения нового товара на рынок благодаря инноваторам и имитаторам.

Решениями уравнений (1) с учетом начального условия $\left.F\right|_{t=0}=0$ и уравнения (2) при начальном условии $\left.F\right|_{t=0}=\alpha$ (параметр $\alpha$ соответствует начальной доле имитаторов от общего числа потребителей) являются функции $F=1-\exp (-p t)$ и $F=\alpha /(\alpha+(1-\alpha) \exp (-q t))$, соответственно.

Согласно моделям Форта-Вудлока и Мэнсфилда диффузия инноваций со временем растет, и протекает она тем интенсивнее, чем выше значения параметров $p$ и $q$ (рис. 2).

Наиболее известной моделью третьей группы является модель Басса [5], в которой учитывается поведение как инноваторов, так и имитаторов. Согласно этой модели, потребление новшеств описывается следующим образом:

$$
\frac{d F}{d t}=p+(q-p) F-q F^{2}
$$

где переменная $F$ и параметры $p$ и $q$ трактуются так же, как и в моделях (1) и (2).

Наиболее важной Басс считал первую группу потребителей новшества - инноваторов, численность которой влияет на распространение информации о новшествах через различные источники коммуникаций, что приводит к увеличению численности представителей второй группы потребителей инноваций имитаторов.

Аналогичные выводы были получены на основе исследований модели Роджерса $[1,7]$. Согласно классификации Е. Роджерса могут быть выделены следующие субъекты - потребители инноваций:

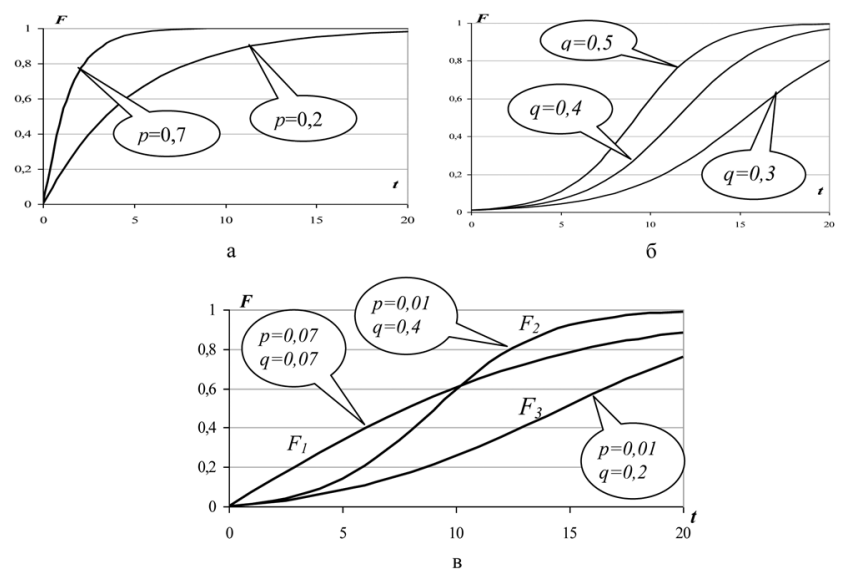

Рис. 2. Геометрическая интерпретация диффузных моделей: $a$ - модель Форта-Вудлока; $\sigma$ - модель

Мэнсфилда (при $\alpha=0,01) ; \beta$ - модель Басса Источник: составлено авторами 
инноваторы, ранние адопторы, раннее большинство, позднее большинство и последователи, на долю которых приходится соответственно 2,5, 13,5, 34, 34 и 16\% потребленных инноваций. В работе [1] было показано, что при наличии достаточного количества ранних потребителей процесс распространения новшеств приобретает необратимый характер, и основное влияние на него оказывают факторы институционального и конкурентного давления. Факторы первой группы повышают конечное потребление инноваций, заставляют инновации становиться социальной нормой, а вторые - повышают спрос на технологии их производства и на новые идеи и изобретения. При этом, очевидно, что и количество последователей инноваторов, как субъектов, способствующих широкому распространению инноваций, имеет существенное значение.

Решением уравнения (3) с учетом начального условия $\left.F\right|_{t=0}=0$ является функция

$$
F=(1-\exp (-(p+q) t)) /(1+(q / p) \exp (-(p+q) t)) .
$$

В модели Басса ввиду того, что используются два параметра, вывод об интенсивности роста переменной $F$ в зависимости от значений параметров $p$ и $q$ не является столь очевидным как в моделях Форта-Вудлока и Мэнсфилда (рис. 2). Чем больше значение одного из параметров при фиксированном значении другого, тем выше интенсивность потребления новшества. Аналогичный вывод однозначно следует, если значения и параметра $p$, и параметра $q$ в одном случае больше, чем в другом. Однако при рассмотрении произвольных комбинаций значений параметров модели выводы не столь очевидны.

В этой связи помимо значений самих параметров $p$ и $q$ актуальность приобретает исследование их соотношения, в качестве которого целесообразно принять отношение $q / p$. Например, для комбинаций значений параметров, представленных на рис. 2, в, это отношение составляет 1 при $p=0,07$ и $q=0,07,20$ при $p=0,01$ и $q=0,2$ и 40 при $p=0,01$ и $q=0,4$. Приведенные данные свидетельствуют, что обособленный анализ параметров $p$ и $q$ и их отношения не позволяет сделать однозначный вывод о том, в каком случае рост функции $F$ будет максимальным. Так, сравнение значений функций $F_{1}, F_{2}$ и $F_{3}$ (рис. $2, \varepsilon$ ) показало, что у функции $F_{1}$ параметр $p$ самый большой, но, начиная с некоторого момента времени, она растет медленнее, чем функция $F_{2}$, у которой параметр $q$ больше, а большие значения соотношения $q / p$ и параметра $q$ у функции $F_{3}$ по сравнению с функцией $F_{1}$ не обеспечивают ей больший рост.

В своих исследованиях [5] Басс установил, что соотношение параметров $q / p$ для 11 видов инновационных товаров меняется в диапазоне от 9,0 до 82,4. По сути, отношение $q / p$ показывает во сколько раз степень проникновения новшеств благодаря имитаторам выше, чем благодаря инноваторам. Таким образом, анализ величины $q / p$ может способствовать пониманию качественного состояния сферы распространения новшеств, что, в свою очередь, дополнит количественные оценки числа пользователей им, рассчитываемые на основе модели (1).
В модели Роджерса аналогом величины $q / p$ выступает обратное отношение количества инноваторов к численности совокупности остальных потребителей инноваций: $97,5 \% / 2,5 \%=39$; т. е. численность потребителей инноваций второй и последующих волн должна в 39 раз превышать численность инноваторов, что согласуется с исследованиями, проведенными Бассом.

Таким образом, из анализа результатов исследований классиков диффузных моделей инноваций следуют количественные оценки, имеющие вполне логичные объяснения, которые могут служить ориентиром при проведении различных исследований.

\section{Результаты и их обсуждение}

Именно с этих позиций авторами были проведены исследования по оценке распространения социальных инноваций в Российской Федерации на основе приведенных выше показателей (табл. 1). Основная сложность при непосредственном определении параметров модели Басса вытекает из незначительной глубины имеющихся данных, что не позволяет использовать классические математико-статистические методы для определения параметров $p$ и $q$. По этой причине основным инструментом определения точного вида модели Басса стала методика, основанная на применении подхода Л. В. Канторовича для определения областей неопределенности искомых параметров [2]. Использование данной методики позволяет уже на стадии моделирования учесть важные требования, оказывающие влияние на точность и адекватность модели. В качестве таких требований были включены условия на априорные минимальные значения параметров $p$ и q и верхний предел средней ошибки аппроксимации ${ }^{2} A_{\text {ср }}$, что позволило выписать следующую задачу для определения параметров модели Басса:

$$
\begin{aligned}
& \varepsilon \rightarrow \min _{p, q}, \\
& \left|\Delta F^{\text {эксп }}\right|_{t}-\left.\Delta F^{\text {расч }}\right|_{t} \mid \leq \varepsilon, t=\overline{1, m}, \\
& p \geq 0,0001 \text {, } \\
& q \geq 0,0001 \text {, } \\
& A_{\text {cp }} \leq 0,4 \text {, }
\end{aligned}
$$

где $t-$ номер наблюдения; $m-$ общее число наблюдений; $\varepsilon$ - предельно допустимая погрешность аппроксимации, представляющая собой максимально возможную величину, на которую могут отличаться

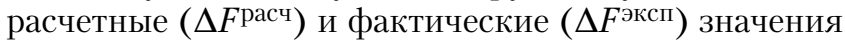
приращений моделируемой величины.

Условие $A_{\text {ср }} \leq 0,4$ фактически определяет в качестве приемлемого 40\%-ный уровень погрешности аппроксимации исходных данных, что, конечно же, не может обеспечить высокую точность модели. Однако в условиях ограниченности исходных данных данный выбор можно считать оправданным.

Анализ результатов проведенных расчетов (табл. 2) выявил низкую инновационную активность

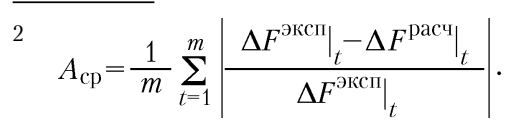


Оптимальные решения задачи (4)

\begin{tabular}{|l|c|c|c|c|c|c|}
\hline Показатель & $p$ & $q$ & $\varepsilon$ & $A_{\text {ср }}$ & $F_{2017}$ & $q / p$ \\
\hline $\begin{array}{l}\text { Количество } \\
\text { социальных } \\
\text { предпринима- } \\
\text { телей, чел. }\end{array}$ & 1,582 & 0,00012 & 3738,2 & 0,22 & 16746,8 & 0,000078 \\
\hline $\begin{array}{l}\text { Количество } \\
\text { запущенных } \\
\text { социально- } \\
\text { предпринима- } \\
\text { тельских } \\
\text { проектов, } \\
\text { проектов }\end{array}$ & 2,482 & 0,00040 & 1917,8 & 0,37 & 5282,2 & 0,000162 \\
\hline $\begin{array}{l}\text { Количество } \\
\text { центров } \\
\text { инноваций в } \\
\text { социальной } \\
\text { сфере, ед. }\end{array}$ & 1,348 & 0,08559 & 1,0 & 0,20 & 18,0 & 0,063486 \\
\hline
\end{tabular}

Источник: составлено авторами

имитаторов, о чем свидетельствуют малые значения отношения $q / p$ для всех исследуемых показателей. При этом прогнозные значения показателей «количество социальных предпринимателей, чел.» и «количество центров инноваций в социальной сфере, ед.» на 2017 г., показывающие тенденцию к положительной динамике развития сферы социальных инноваций в России, следует рассматривать как достоверные в силу относительно небольших значений средней ошибки аппроксимации $A_{\text {cp }}$.

\section{Заключение}

Очевидно, что точность оценок параметров $p$ и $q$, равно как и достоверность прогнозных оценок, будет увеличиваться по мере накопления статистических данных. Однако даже в условиях ограниченности информации представленный подход к моделированию тенденций распространения социальных инноваций позволяет получить количественные оценки происходящих в данной сфере процессов, в рамках как ретроспективного, так и перспективного анализов, что может стать основой для корректировки стратегии развития сферы социальных инноваций в части тиражирования и масштабирования социальных инновационных проектов, степени заимствования положительного опыта, а также динамики увеличения благополучателей указанных проектов.

\section{Список использованных источников}

1. Р. Грант. Современный стратегический анализ. СПб.: Питер, 2008. - 560 c

2. О. Г. Кантор. Оценка параметров диффузных процессов в сфере инноваций Российской Федерации//Проблемы теории и практики управления. № 5. 2015. С. 93-99.

3. В. М. Полтерович. Проблема формирования национальной инновационной системы//Экономика и математические методы. № 2. 2009. С. 3-18.

4. Официальный сайт Агентства стратегических инициатив. http://asi.ru.

5. M. Bass Frank. A New Product Grows for Model Consumer Durables//Management Science. V. 15. No. 5. 2004. P. 215-227.

6. L. F. Fourt, J. W. WoodLock. Early prediction of Market success for new grocery products//Journal of Marketing. Vol. 26. No. 2. 1960. P. 31-38.

7. E. M. Rogers. Diffusion of Innovations (5th ed.). New York: Free Press, 2003. $-576 \mathrm{p}$.

Characteristics' Evaluation of the Dissemination of Social Innovations Process in the Russian Federation O. G. Kantor, PhD in Physics and Mathematics, Associate Professor, Senior scientist.

Yu. A. Kuznetsova, PhD in Economics, Senior scientist.

(Institute of Social and Economic Research Ufa Scientific Centre Russian Academy of Sciences)

The concept of «social innovation" is relatively new in Russia in terms of theoretical or methodological design, and also practical use. The paper presents the results of studies on the propagation characteristics of social innovation, conducted from the standpoint of traditional and specific approaches. It has been shown that in the Russian Federation's current environment the spread of social innovation is of «viscous» behavior, which manifests itself, in particular, by the limited possible spread of innovations and their appropriate scaling. Practical steps taken to regulate the spread of social innovations have led to the foundation of the Social Initiatives Agency - the organization that accumulates social initiatives, determines goals and priorities for the development and spread of social innovations. However, given the novelty of this concept in the country, development of specific methods that allow to study innovation processes in the social sector comes to be of special importance. Implementation of such methods will contribute to a comprehensive analysis and will improve the assessments validity. One approach to the implementation of the above is to use the concept of innovation diffusion, within the framework of which the authors proposed a method for determining the parameters of the Bass model, carried out the appropriate calculations and interpreted the obtained results.

Keywords: social innovation, innovative project, dissemination, evaluation, diffusion, innovator, imitator. 